Cite this: Analyst, 2013, 138, 7326

Received 12th July 2013

Accepted 8th October 2013

DOI: $10.1039 /$ c3an01339a

www.rsc.org/analyst

\section{Fluorogenic probes using 4-substituted-2- nitrobenzenesulfonyl derivatives as caging groups for the analysis of human glutathione transferase catalyzed reactions $\uparrow$}

\author{
Aya Shibata, ${ }^{\text {ah }}$ Yukiko Nakano, ${ }^{\text {ah }}$ Mika Ito, ${ }^{\text {abh }}$ Mika Araki, ${ }^{\text {ach }}$ Jie Zhang, ${ }^{f}$ \\ Yasuhiko Yoshida, ${ }^{c}$ Satoshi Shuto, ${ }^{b}$ Bengt Mannervik, ${ }^{\text {de }}$ Ralf Mogenstern, ${ }^{f}$ \\ Yoshihiro Ito*ah and Hiroshi Abe*abgh
}

\begin{abstract}
We have synthesized a series of 4-substituted-2-nitrobenzene-sulfonyl compounds for caged fluorogenic probes and conducted a Hammett plot analysis using the steady-state kinetic parameters. The results revealed that the glutathione transferase (GST) alpha catalyzed reaction was dependent on the $\sigma$ value in the same way as the non-enzymatic reaction, whereas the dependence of the $\sigma$ value of the GST mu and pi was not as pronounced as that of GST alpha.
\end{abstract}

Glutathione transferases (GSTs) are phase II enzymes that catalyze the nucleophilic attack of glutathione (GSH) to a wide range of hydrophobic and electrophilic compounds. ${ }^{1-3}$ The substrates for these enzymes include a number of chemical carcinogens, therapeutic drugs, and lipid peroxidation products. Different GST enzyme families exist in the cytosol and in membranes. Human cytosolic GSTs ${ }^{\mathbf{4 , 5}}$ are now grouped into at least eight independent classes, including alpha, kappa, mu, pi, sigma, theta, zeta and omega, whereas human microsomal GSTs $^{6,7}$ are grouped into the membrane-associated proteins of the eicosanoid and glutathione metabolism (MAPEG) superfamily, which now includes microsomal GST1 (MGST1), MGST2, MGST3, leukotriene $\mathrm{C}_{4}$ synthase, microsomal prostaglandin $\mathrm{E}_{2}$ synthase 1 and 5-lipoxygenase activating protein. GSTs are often overexpressed in a wide variety of tumors

${ }^{a}$ Nano Medical Engineering Laboratory, RIKEN, 2-1 Hirosawa, Wako-Shi, Saitama 351-0198, Japan. E-mail: y-ito@riken.jp; Fax: +81-48-462-9300; Tel: +81-48-467-4979 ${ }^{b}$ Faculty of Pharmaceutical Sciences, Hokkaido University, Kita-12, Nishi-6, Kita-ku, Sapporo-Shi, Hokkaido 060-0812, Japan.E-mail: h-abe@pharm.hokudai.ac.jp ${ }^{c}$ Graduate School of Engineering, Toyo University, 2100 Kujirai, Kawagoe-Shi, Saitama 350-8585, Japan

${ }^{d}$ Department of Biochemistry, Uppsala University, Biomedical Center, Box 576, SE-75123 Uppsala, Sweden

${ }^{e}$ Department of Neurochemistry, Stockholm University, Svante Arrhenius väg 21A, SE-10691 Stockholm, Sweden

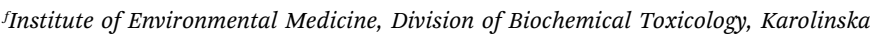
Institutet, SE-17177 Stockholm, Sweden

${ }^{g}$ PRESTO, Japan Science and Technology Agency, 4-1-8, Honcho, Kawaguchi-Shi, Saitama 332-0012, Japan

${ }^{h}$ Emergent Bioengineering Materials Research Team, RIKEN Center for Emergent Matter Science, 2-1 Hirosawa, Wako-shi, Saitama 351-0198, Japan

$\uparrow$ Electronic supplementary information (ESI) available. See DOI: 10.1039/c3an01339a compared with the normal tissue and are involved in the development of resistance to various anticancer drugs. ${ }^{8-10}$

Studies aimed at measuring GST activity have traditionally been performed using the chromogenic substrate 1-chloro-2,4dinitrobenzene (CDNB). ${ }^{11}$ Unfortunately, however, the available change in absorption at $340 \mathrm{~nm}$ is often limited by the low sensitivity and selectivity of this substrate, and some GSTs have low activity with CDNB. As a consequence, several groups have developed fluorogenic ${ }^{12-16}$ and bioluminogenic ${ }^{17}$ probes to provide higher levels of sensitivity for the detection of GST activity. We previously reported the development of a general strategy for the synthesis of probes for the detection of GSTs, including fluorogenic, ${ }^{18,19}$ bioluminogenic ${ }^{20}$ and ${ }^{19} \mathrm{~F}^{-\mathrm{NMR}^{20}}$ probes. Arylsulfonyl groups, such as 2,4-dinitrobenzenesulfonamide (DNs), were used as caging groups to design these probes. GST catalyzes the nucleophilic aromatic substitution $\left(\mathrm{S}_{\mathrm{N}} \mathrm{Ar}\right)$ of GSH to the $\alpha$-carbon of the arylsulfonyl group via a Meisenheimer complex. This process leads to the release of the uncaged molecule, which generates a specific signal. In general, Meisenheimer intermediates are stabilized by electron-withdrawing groups conjugated with the aromatic ring. To date, the effects of electron-withdrawing groups on $\mathrm{S}_{\mathrm{N}} \mathrm{Ar}$ reactions catalyzed by rat GSTs have been examined using several 2- or 4-substituted CDNB analogs, and a linear correlation was reported between the reactions catalyzed by the rat GSTs and the Hammett substituent constant $\left(\sigma^{-}\right) \cdot{ }^{21-25}$ For the 2-substituted CDNB analogs, ${ }^{23,24}$ the catalysis of the reaction by GST1-1 and GST7-7 was found to be dependent on the $\sigma$ value in the same way as the non-enzymatic reaction. In contrast, the reactions catalyzed by GST3-3 and GST4-4 were independent of the $\sigma$ value. A correlation has been reported previously for the GST4-4 and MGST1 catalyzed reactions of the 4-substituted CDNB analogs. ${ }^{22,25,26}$ Clearly, GST catalyzed reactions can be influenced 
by the electron-withdrawing capacity of the substituents attached to the phenyl ring. In the current paper, we have examined the catalytic capacity of the main human cytosolic GSTs (i.e., alpha, $\mathrm{mu}$ and pi) towards the GSH conjugation reactions of a series of 4-substituted-2-nitrobenzenesulfonyl group caged mono-acetyl rhodamine (AcRh) compounds. Furthermore, the probes were investigated regarding optimal properties for the detection of intracellular GST activity.

The structures of the compounds from the 4-substituted-2nitrobenzenesulfonyl group protected AcRh series are shown in Fig. 1 with DNs-caged AcRh (DNs-AcRh), 4-cyano-2-nitro-benzenesulfonyl (CNs)-caged AcRh (CNs-AcRh), 4-acetyl-2-nitrobenzenesulfonyl (ANs)-caged AcRh (ANs-AcRh) and 4-butyl ester-2-nitro-benzenesulfonyl (BNs)-caged AcRh (BNs-AcRh). The synthesis of the DNs-AcRh compounds was described in our previous paper. ${ }^{19}$ A series of 4-substituted-2-nitrobenzenesulfonyl chlorides were prepared via the thiol compound from the corresponding 4-substituted-1-chloro-2nitrobenzene. ${ }^{27}$ The desired benzene sulfonamide-protected rhodamine derivatives (CNs-AcRh, ANs-AcRh and BNs-AcRh) were obtained from AcRh following its treatment with the corresponding 4-substituted-2-nitrobenzenesulfonyl chlorides in a mixture of pyridine and $\mathrm{CH}_{2} \mathrm{Cl}_{2}$ for $16-21 \mathrm{~h}$, with yields of 84 , 94 , and $54 \%$, respectively. The probes showed very low fluorescence quantum yields (i.e., 0.008-0.044) compared with that of AcRh, which was much higher at 0.387. The observed fluorescence signal was therefore derived from the AcRh following its deprotection, which was catalyzed by GST.

We initially examined whether these sulfonamides could be cleaved by the GST-catalyzed reaction using commercially available human GST, which is a mixture of GST alpha and mu. The probes were incubated in PBS buffer ( $\mathrm{pH}$ 7.4) containing $1 \mathrm{mM} \mathrm{GSH}$ and $2 \mu \mathrm{g} \mathrm{mL}{ }^{-1}$ recombinant GST for $30 \mathrm{~min}$ at $37^{\circ} \mathrm{C}$. The fluorescence signal derived from the emission at $522 \mathrm{~nm}$ was monitored over time and the conversion yield was calculated from the authentic sample (Fig. S1 $\dagger$ ). The fluorescence signals increased with time for all of the probes following the deprotection of the caged group to the amino group of the AcRh. In the case of DNs-AcRh, the reaction reached $80 \%$ conversion after 6 min and reached the saturation phase. In contrast, the

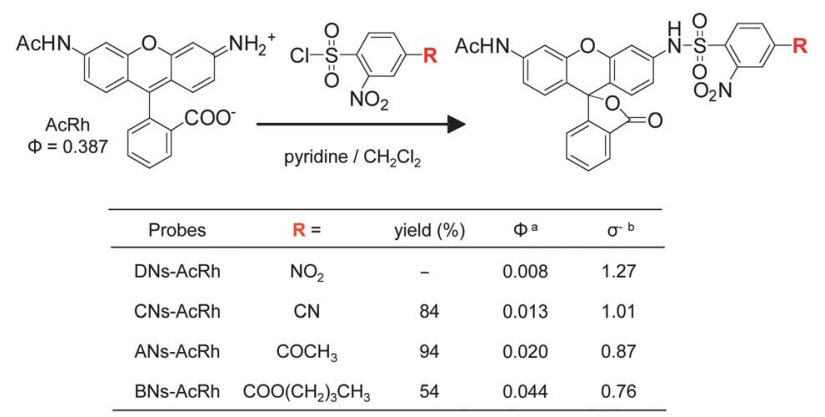

Fig. 1 Synthesis and quantum yields of 4-substituted-2-nitrobenzenesulfonyl protected AcRh probes. a All measurements were performed in $\mathrm{PBS}(\mathrm{pH} 7.4$, $10 \mathrm{mM}$ ). The compounds were excited at $490 \mathrm{~nm}$, and the quantum yields were determined using fluorescein $(\Phi=0.85,0.1 \mathrm{M} \mathrm{NaOH})$ as a standard. ${ }^{\mathrm{b}}$ The Hammett constants $\left(\sigma^{-}\right)$were taken from ref. 24 and 28. reaction of BNs-AcRh was much slower than that of DNs-AcRh, with the reaction only reaching $16 \%$ conversion after $30 \mathrm{~min}$. Based on these results, the rate of the deprotection appeared to be dependent on the values of the substituent constant ${ }^{28}$ of the 4-substituent group.

We then performed the steady-state kinetic analysis of the DNs-, CNs-, ANs- and BNs-AcRh with three representative cytosolic GSTs, including GSTA1-1, GSTM2-2 and GSTP1-1. The steady-state kinetic parameters are shown in Table 1 . None of the probes were selective for a specific GST isozyme. The $k_{\text {cat }} / K_{\mathrm{m}}$ values for GSTA1-1 were greater than those of GSTM2-2 and P1-1 for the deprotection reactions of all the probes studied. The rate enhancement was $10^{6}$ to $10^{4}$ and remained lower than the values previously reported. ${ }^{19}$ The $K_{\mathrm{m}}$ values of BNs-AcRh for all of the GSTs were lower than those of the other probes. The substrate binding sites of GSTs, which are known as H-sites, contain a large number of hydrophobic amino acid residues. ${ }^{29}$ The BNs group has a long lipophilic alkyl chain, and the high affinity of the BNs-AcRh towards the GSTs basically reflects the strong hydrophobic interaction between the lipophilic butyl ester group and hydrophobic amino acid residues. Hammett plots were constructed from these kinetic parameters to investigate the relationship between the GST catalyzed reaction and the nature of the electron-withdrawing substituents. These Hammett plots are shown in Fig. 2 and S2. $\dagger$

In the Hammett plots for $K_{\mathrm{m}}$, no correlations were observed between $K_{\mathrm{m}}$ and $\sigma^{-}$for any of the GSTs (Fig. S2A $\dagger$ ). This result was consistent with the previously reported data using 2 -substituted-CDNB analogs. ${ }^{24}$ With regard to $k_{\text {cat }}$, the results revealed a correlation with the substituent constants $(r=0.758$, 0.727 and 0.808 ; Fig. $\mathrm{S} 2 \mathrm{~B} \dagger$ ). In this case, only the $k_{\text {cat }}$ value of the BNs-AcRh was found to deviate from the fitted line. When BNsAcRh was excluded from the fitting, the correlation coefficient improved to $r>0.9$. The butyl ester represents a relatively long alkyl chain compared with the other substituent groups, and the steric restriction imposed by this group may have influenced the $k_{\text {cat }}$ value. It is therefore necessary to change the butyl ester group to a shorter substituent group, such as a methyl ester, and further examine the relationship between $k_{\text {cat }}$ and the $\sigma^{-}$value. In Fig. 2, the Hammett plot indicates a good correlation coefficient between the $\log k_{\text {non-cat }}$ values of the nonenzymatic reaction and $\sigma^{-}(r=0.93)$. For the GST-catalyzed reaction, the linear plot also gave a good correlation coefficient for the relationship between $\log k_{\text {cat }} / K_{\mathrm{m}}$ and $\sigma^{-}(r>0.9)$, and the following Hammett equations were obtained:

$$
\begin{aligned}
\text { No GST: } \log \left(k_{\text {non-cat }}\right)= & (2.22 \pm 0.11) \sigma^{-}-(1.90 \pm 0.08) \\
& (r=0.930, s=0.238, n=4) \\
\text { GSTA1-1: } \log \left(k_{\text {cat }} / K_{\mathrm{m}}\right)= & (2.05 \pm 0.04) \sigma^{-}+(4.29 \pm 0.05) \\
& (r=0.932, s=0.238, n=4) \\
\text { GSTM2-2: } \log \left(k_{\text {cat }} / K_{\mathrm{m}}\right)= & (0.90 \pm 0.12) \sigma^{-}+(4.61 \pm 0.14) \\
& (r=0.945, s=0.084, n=4) \\
\text { GSTP1-1: } \log \left(k_{\mathrm{cat}} / K_{\mathrm{m}}\right)= & (0.83 \pm 0.13) \sigma^{-}+(4.24 \pm 0.15) \\
& (r=0.959, s=0.066, n=4)
\end{aligned}
$$


Table 1 Steady-state kinetic parameters of GST with 4-substituted-2-nitrobenzenesulfonyl protected AcRh ${ }^{2}$

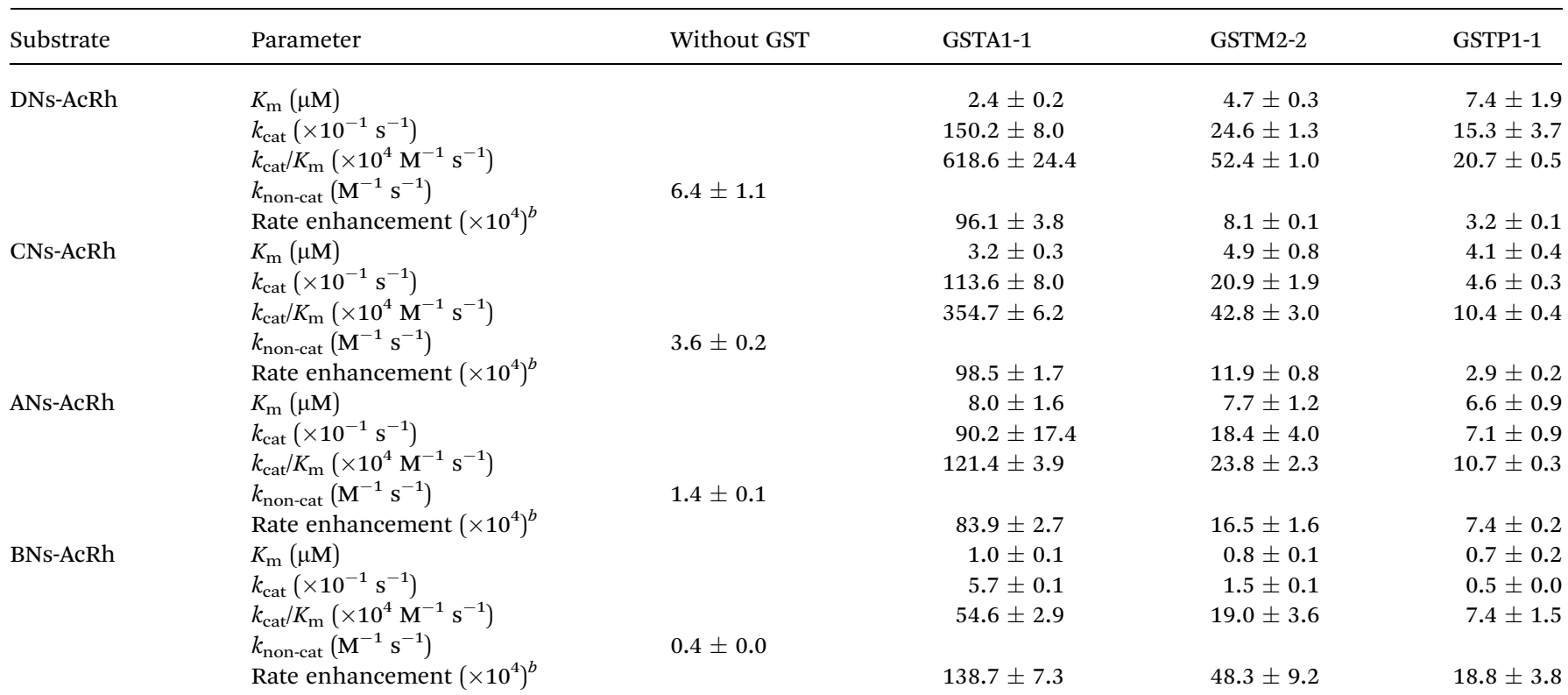

${ }^{a}$ The GST (GSTA1-1, GSTM2-2, or GSTP1-1) was assayed with the constant GSH at $5 \mathrm{mM}$ in $0.1 \mathrm{M}$ potassium phosphate buffer (pH 6.5) while varying the concentration of DNs-AcRh $(1-10 \mu \mathrm{M})$, CNs-AcRh $(1-10 \mu \mathrm{M})$, ANs-AcRh $(1-30 \mu \mathrm{M})$, or BNs-AcRh $(1-50 \mu \mathrm{M})$. The final concentration of the DMSO solvent was $1 \%$. The values are the means \pm standard errors of the mean $(n=3) .{ }^{b}$ Rate enhancement $=\left(k_{\text {cat }} / K_{\mathrm{m}}\right) / k_{\text {non-cat }}$.

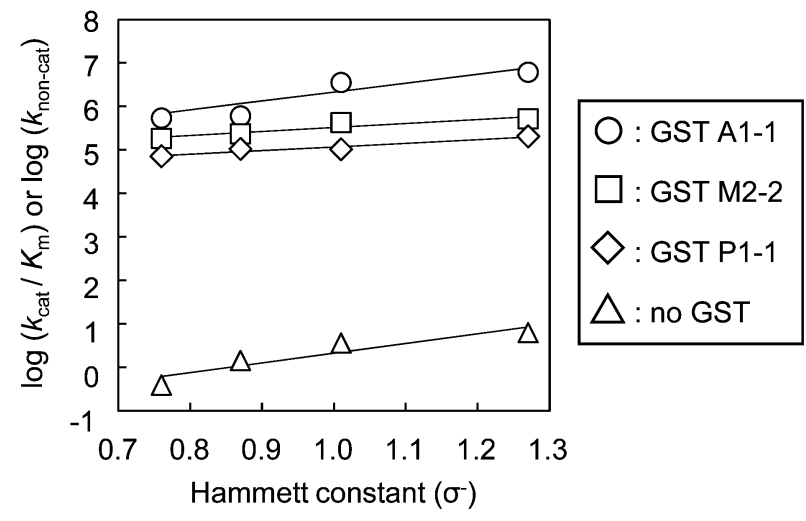

Fig. 2 Hammett plot of $\log \left(k_{\text {cat }} / K_{m}\right)$ or $\log \left(k_{\text {non-cat }}\right)$ versus $\sigma^{-}$for non-enzymatic or enzyme-catalyzed reaction. Hammett constants are from Fig. 1 , and $k_{\text {cat }} / K_{\mathrm{m}}$ and $k_{\text {non-cat }}$ values are from Table 1. Lines are least-square fits to the data points by Microsoft Excel.

The $\rho$ value for the non-enzymatic reaction $(\rho=2.22$, eqn (1)), which was a general $S_{N} A r$ reaction, was large because the electronic nature of the substituent had a significant impact on the rate of the $\mathrm{S}_{\mathrm{N}} \mathrm{Ar}$ reaction. For GSTA1-1, the $\rho$ value was comparable to that of the non-enzymatic reaction $(\rho=2.05$, eqn (2)). This result indicated that the rate-limiting step of the GSTA1-1 catalyzed-deprotection involved the formation of a Meisenheimer complex in the same way as a general $\mathrm{S}_{\mathrm{N}} \mathrm{Ar}$ reaction. In contrast, the slopes for GSTM2-2 and P1-1 were very low compared with the non-enzymatic reaction, and the $\rho$ values were 0.90 and 0.83 , respectively (eqn (3) and (4)). These results indicated that the presence of an electron-withdrawing group did not have a significant impact on the GSTM2-2 and P1-1 catalyzed-reactions. X-ray crystallographic analysis of GST pi and $\mathrm{mu}$ in the complex with a transition state analog revealed that two tyrosine residues formed hydrogen bonding interactions with the 2-nitro group of the analog. ${ }^{30}$ Since the Meisenheimer complex was stabilized by tyrosine residues, it was envisaged that changes in this substituent group would have very little impact on the reaction.

Multiple regression analysis was performed for $\log k_{\text {non-cat }}$ or $\log k_{\text {cat }} / K_{\mathrm{m}}$ using a series of physicochemical parameters (Fig. S3 $\uparrow$ ). When multiple regressions were performed using $\sigma^{-}$ and the field parameter $(F)$, the correlation coefficient for GSTP1-1 improved substantially from 0.959 to 0.992 . This result indicated that the field effect, which induces the polarization of the $\sigma$-bonds in the GSTP1-1 catalyzed reaction, had a major influence on $k_{\text {cat }} / K_{\mathrm{m}}$ and contributed to the value in a negative manner. When the fitting was performed using $F$ and the resonance parameter $(R)$, there was a clear increase in the correlation coefficient $(r>0.99)$ for all of the GSTs, except for GSTP1-1. This result indicated that resonance effects, which contribute to the stabilization of the $\pi$ electrons of the aromatic ring, did not have an impact on the GSTP1-1 catalyzed reaction. The correlation coefficient for the $F$ value of the GSTM2-2 reaction was small compared with the corresponding coefficients of the non-enzymatic and GSTA1-1 reactions. This result suggested that the substituent at the para position may undergo some interactions with amino acid residues within GSTM2-2. The correlation coefficients for the $R$ values of the GSTA1-1 and GSTM2-2 reactions were small compared with that of the nonenzymatic reaction. This result showed that the contribution of the resonance effect was smaller than that of the non-enzymatic reaction for the GST catalyzed reactions. When the hydrophobic fragmental constants $(f)$, which provide some indication of the 


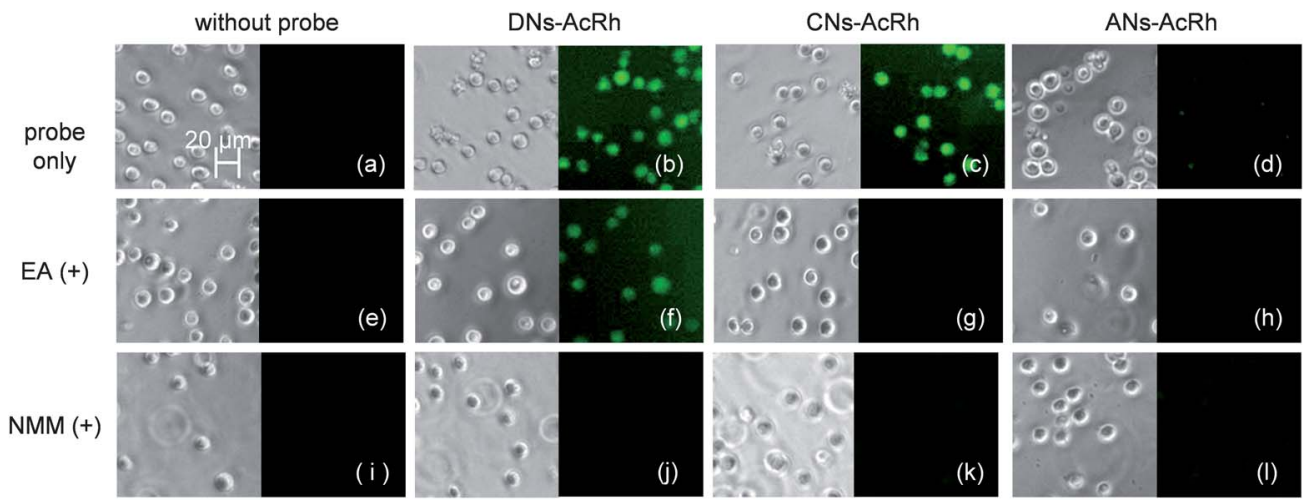

Fig. 3 Fluorescence images of the $\mathrm{HL}-60$ cells: (a-d) images of the cells incubated with a $10 \mu \mathrm{M}$ probe for 60 min at $37^{\circ} \mathrm{C}$. (e-I) control images of the cells pretreated with $50 \mu \mathrm{M} \mathrm{EA}(\mathrm{e}-\mathrm{h})$ or $1 \mathrm{mM} \mathrm{NMM}(\mathrm{i}-\mathrm{l})$ for $60 \mathrm{~min}$ at $37^{\circ} \mathrm{C}$ before being incubated with a $10 \mu \mathrm{M}$ probe for $60 \mathrm{~min}$ at $37^{\circ} \mathrm{C}$. (left panel) Bright-field image; (right panel) fluorescence image. Microscope settings were as follows: excitation: 470/40 bandpass filter; emission: 525/50 bandpass filter.

lipophilicity of the substituents, were used together with $\sigma^{-}$for the fitting, there was no clear increase in the correlation coefficient for the GSTM2-2 or GSTP1-1 catalyzed reaction. This result indicated that the lipophilicities of the substituents did not have a significant impact on the GSTM2-2 reaction, and that the value of GSTP1-1 was lower than that of GSTA1-1. The Sterimol parameters $\left(L\right.$ and $\left.B_{5}\right)$ were used to investigate the influence of any steric effects on the reaction. In the nonenzymatic and the GSTA1-1 catalyzed reaction, when $B_{5}$ was used for the analysis with $\sigma^{-}$, the correlation coefficient increased clearly $(r>0.99)$. In the GSTM2-2 and GSTP1-1 catalyzed reactions, when $L$ or $B_{5}$ was added as the variable, the correlation coefficient was almost the same as the value of $\sigma^{-}$ alone. These results suggested that the GSTM2-2 and GSTP1-1 catalyzed reactions were not easily influenced by steric effects, whereas the GSTA1-1 catalyzed reaction was sensitive to the influence of the steric effect.

Finally, we examined the imaging of GST activity in living cells using DNs-AcRh, CNs-AcRh, and ANs-AcRh. HL-60 cells, which predominantly express GSTP1-1 and GSTM2-2, ${ }^{31}$ were selected as the imaging cells. We imaged cells treated with $\mathrm{N}$-methylmaleimide (NMM), which is a known blocking reagent for thiols such as GSH, and ethacrynic acid (EA), ${ }^{32}$ which is a known inhibitor of GSTs, as controls. The fluorescence images recorded after 60 min are shown in Fig. 3. When the cells were treated with the probe in isolation, the fluorescence signals of the cells were found to be dependent on the electronic nature of the substituent group. DNs-AcRh and CNs-AcRh showed strong fluorescence signals, whereas the fluorescence intensity of the ANs-AcRh signal was particularly weak. When the cells were treated with EA, CNs-AcRh and ANs-AcRh did not provide any fluorescence signals. In contrast, DNs-AcRh gave a clear fluorescence signal under the same conditions. In the cytoplasm, GSH exists in the millimolar concentration range. ${ }^{33}$ In the test tube, we treated the probes with $1 \mathrm{mM} \mathrm{GSH}$ and monitored the resulting fluorescence signals for $60 \mathrm{~min}$ (Fig. S4 †). DNs-AcRh was deprotected in a $33 \%$ yield. Under the same conditions, the other probes remained largely unchanged, with only small quantities of AcRh being formed. Furthermore, when the cells were treated with NMM and depleted of GSH, none of the probes provided any fluorescence signals as expected. The results from the inhibitor experiment revealed that DNs-AcRh reacted with intracellular thiols also independent of GST and generated a nonspecific fluorescence signal. On the basis of these results, it was concluded that CNs-AcRh represents the best probe for the detection of GST activity in living cells.

\section{Conclusions}

We have investigated the influence of the substituent constant on the human GST catalyzed-reaction using several 4-substituted-arylsulfonyl group caged fluorogenic probes and demonstrated that the different GST subtypes respond differently to probes with varied electron-withdrawing groups. Furthermore, cell imaging experiments revealed that the CNsprotected probe was the best probe for the detection of GST activity. These results could provide a platform for the design of new GST probes and define conditions for live cell GST imaging.

\section{Acknowledgements}

H.A. was financially supported by Strategic International Research Cooperative Program (SICORP) from Japan Science and Technology Agency (JST). A.S. was financially supported by a Grant-in-Aid for Young Scientists (B) (Grant no. 22790124) and the Special Postdoctoral Researcher Program of RIKEN. B.M. was supported by the Swedish Research Council and the Swedish Cancer Society. R.M. was supported by the Swedish Research Council, the Swedish Foundation for Strategic Research, VINNOVA and funds from Karolinska Institutet. J.Z. was financially supported by the Swedish Research Council (No. 524-2011-6998).

\section{Notes and references}

1 L. F. Chasseaud, Adv. Cancer Res., 1979, 29, 175-274.

2 W. H. Habig, M. J. Pabst and W. B. Jakoby, J. Biol. Chem., 1974, 249, 7130-7139.

3 B. Mannervik, Adv. Enzymol. Relat. Areas Mol. Biol., 1985, 57, 357-417.

4 J. D. Hayes and D. J. Pulford, Crit. Rev. Biochem. Mol. Biol., 1995, 30, 445-600. 
5 R. N. Armstrong, Chem. Res. Toxicol., 1997, 10, 2-18.

6 R. Rinaldi, E. Eliasson, S. Swedmark and R. Morgenstern, Drug Metab. Dispos., 2002, 30, 1053-1058.

7 P. J. Jakobsson, R. Morgenstern, J. Mancini, A. FordHutchinson and B. Persson, Protein Sci., 1999, 8, 689-692.

8 G. Jedlitschky, I. Leier, U. Buchholz, M. Center and D. Keppler, Cancer Res., 1994, 54, 4833-4836.

9 J. A. Moscow, C. R. Fairchild, M. J. Madden, D. T. Ransom, H. S. Wieand, E. E. O'Brien, D. G. Poplack, J. Cossman, C. E. Myers and K. H. Cowan, Cancer Res., 1989, 49, 1422-1428.

10 J. Ping, H. Wang, M. Huang and Z. S. Liu, Toxicol. Sci., 2006, 89, 438-443.

11 U. H. Danielson and B. Mannervik, Biochem. J., 1988, 250, 705-711.

12 B. S. Nieslanik and W. M. Atkins, J. Biol. Chem., 2000, 275, 17447-17451.

13 R. Morgenstern, J. W. DePierre and L. Ernster, Biochem. Biophys. Res. Commun., 1979, 87, 657-663.

14 R. Nauen and N. Stumpf, Anal. Biochem., 2002, 303, 194-198. 15 R. Svensson, C. Greno, A. S. Johansson, B. Mannervik and R. Morgenstern, Anal. Biochem., 2002, 311, 171-178.

16 Y. Fujikawa, Y. Urano, T. Komatsu, K. Hanaoka, H. Kojima, T. Terai, H. Inoue and T. Nagano, J. Am. Chem. Soc., 2008, 130, 14533-14543.

17 W. Zhou, J. W. Shultz, N. Murphy, E. M. Hawkins, L. Bernad, T. Good, L. Moothart, S. Frackman, D. H. Klaubert, R. F. Bulleit and K. V. Wood, Chem. Commun., 2006, 46204622.

18 J. Alander, K. Johansson, V. D. Heuser, H. Farebo, J. Jarvliden, H. Abe, A. Shibata, M. Ito, Y. Ito and R. Morgenstern, Anal. Biochem., 2009, 390, 52-56.

19 J. Zhang, A. Shibata, M. Ito, S. Shuto, Y. Ito, B. Mannervik, H. Abe and R. Morgenstern, J. Am. Chem. Soc., 2011, 133, 14109-14119.
20 M. Ito, A. Shibata, J. Zhang, M. Hiroshima, Y. Sako, Y. Nakano, K. Kojima-Aikawa, B. Mannervik, S. Shuto, Y. Ito, R. Morgenstern and H. Abe, ChemBioChem, 2012, 13, 1428-1432.

21 J. H. Keen, W. H. Habig and W. B. Jakoby, J. Biol. Chem., 1976, 251, 6183-6188.

22 W. J. Chen, G. F. Graminski and R. N. Armstrong, Biochemistry, 1988, 27, 647-654.

23 E. M. van der Aar, D. Buikema, J. N. Commandeur, J. M. te Koppele, B. van Ommen, P. J. van Bladeren and N. P. Vermeulen, Xenobiotica, 1996, 26, 143-155.

24 E. M. Van der Aar, T. Bouwman, J. N. Commandeur and N. P. Vermeulen, Biochem. J., 1996, 320, 531-540.

25 E. M. van der Aar, M. J. de Groot, T. Bouwman, G. J. Bijloo, J. N. Commandeur and N. P. Vermeulen, Chem. Res. Toxicol., 1997, 10, 439-449.

26 R. Morgenstern, G. Lundqvist, V. Hancock and J. W. DePierre, J. Biol. Chem., 1988, 263, 6671-6675.

27 D. Crich and I. Sharma, Angew. Chem., Int. Ed. Engl., 2009, 48, 7591-7594.

28 C. G. Swain and E. C. Lupton, J. Am. Chem. Soc., 1968, 90, 4328-4337.

29 C. M. Bruns, I. Hubatsch, M. Ridderström, B. Mannervik and J. A. Tainer, J. Mol. Biol., 1999, 288, 427-439.

30 L. Prade, R. Huber, T. H. Manoharan, W. E. Fahl and W. Reuter, Structure, 1997, 5, 1287-1295.

31 K. D. Tew, A. Monks, L. Barone, D. Rosser, G. Akerman, J. A. Montali, J. B. Wheatley and D. E. Schmidt, Jr, Mol. Pharmacol., 1996, 50, 149-159.

32 P. J. O'Dwyer, F. LaCreta, S. Nash, P. W. Tinsley, R. Schilder, M. L. Clapper, K. D. Tew, L. Panting, S. Litwin, R. L. Comis, et al., Cancer Res., 1991, 51, 6059-6065.

33 A. Meister and S. S. Tate, Annu. Rev. Biochem., 1976, 45, 559604. 